\title{
Bilateral Continuous Erector Spinae Plane Blocks for the Management of Post-Thoracosternotomy Pain in Patients Undergoing Lung Transplantation Initial Experience at Toronto General Hospital
}

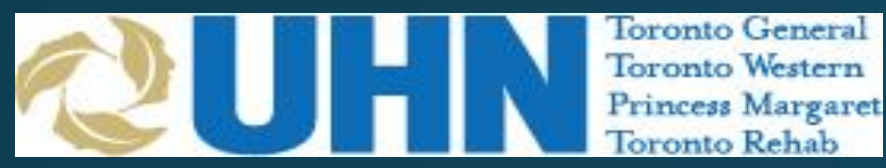

C. Hanley ${ }^{1}$, A. Spring ${ }^{1}$, S. Ladak², J. Jiang ${ }^{2}$, D. Tamir ${ }^{1}$, B. Ansari ${ }^{1}$

${ }^{1}$ Toronto General Hospital, Anesthesia, Toronto, Canada.

${ }^{2}$ Toronto General Hospital, Acute Pain Service, Toronto, Canada.

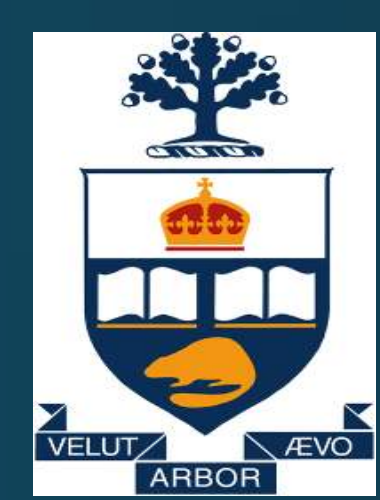

\section{BACKGROUND}

- The management of post-thoracotomy pain is a challenging and contentious issue in patients who undergo lung transplantation.

- Poorly managed acute pain and an over-reliance on opioid analgesia may have a significant adverse impact on respiratory, haemodynamic, cognitive, metabolic and functional outcomes in this vulnerable population. In particular, the impact on respiratory mechanics may ultimately lead to graft dysfunction and prolonged mechanical ventilation.

- Lateral thoracotomy and thoracosternotomy ("clamshell") incisions are associated with significant pain; the latter is considered the most painful.

- Thoracic epidural analgesia (TEA) is considered the gold standard for post-thoracotomy pain, and its benefits in lung transplantation are are well documented.

- However, at our centre, it is frequently precluded by factors such as systemic heparinisation, use of intraoperative venoarterial extracorporeal membrane oxygenation (VA-ECMO), haemodynamic instability, and coagulopathy associated with prolonged surgery and transfusion.

- The erector spinae plane block (ESPB) is a novel ultrasoundguided interfascial plane block which has been shown to provide efficacious analgesia in thoracic surgical patients and in those with chronic thoracic neuropathic pain.

- It targets not only the ventral and dorsal rami of the thoracic spinal nerve roots, but also the rami communicantes, ultimately resulting in somatic and visceral anaesthesia of the ipsilateral hemithorax (Figure 1).

- We postulated that the ESP block could play an important analgesic role as part of a multimodal enhanced recovery regimen for patients undergoing lung transplantation at our centre.

- To the best of our knowledge there has been only 1 case report of its use specifically in lung transplantation.

\section{METHODS}

- We conducted a retrospective case review of the first series of patients who had bilateral ESP blocks placed for management of pain following lung transplantation.

- The primary outcome was opioid PCA consumption within the first 72 hours postoperatively.

- Secondary outcomes included numerical rating "worst pain" scores, functional outcomes e.g. mobilisation distance in the first 72 hours, duration of mechanical ventilation, and length of ICU stay.

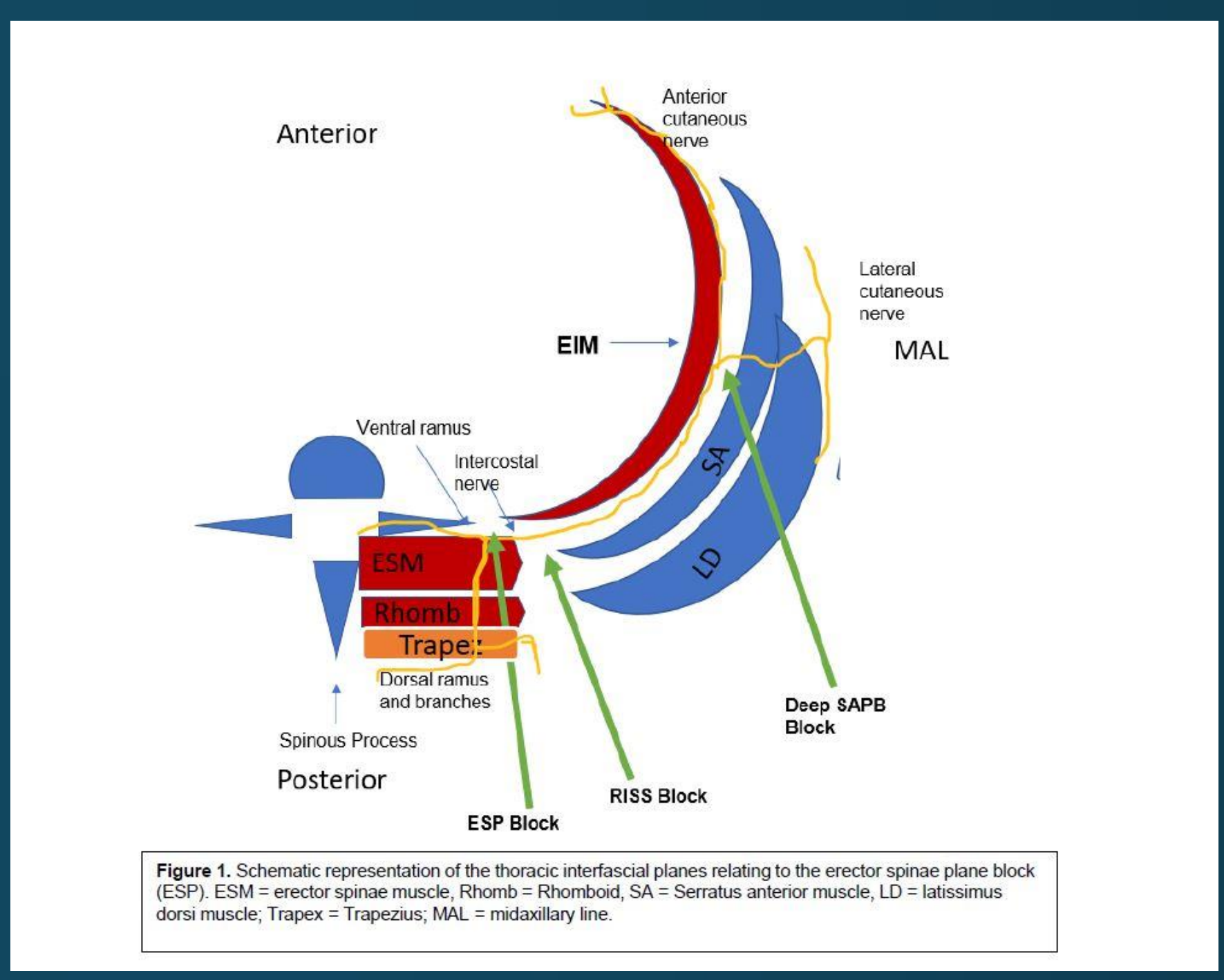

\section{RESULTS}

- We can report the general overall findings of the case series.

- A retrospective chart review was performed; 1 patient was excluded due to missing data.

- All were ASA IV-E, and underwent lung transplantation via bilateral thoracosternotomy

- The majority required intraoperative VA-ECMO.

- $1 / 3$ were on regular preoperative opioids.

- The primary indications were COPD, and idiopathic pulmonary fibrosis/ interstitial lung disease.

- The blocks were performed at the end of surgery in most cases, according to the technique described by Forero et al.

- Opioid requirements were not increased compared with what would have been expected in the absence of the block.

- Worst pain scores were satisfactory, albeit higher in patients who were not opioid naïve, although still in the mild-moderate range.

- There was a low incidence of postoperative delirium

- The majority of patients were extubated within 48 hours

- Mobilisation goals were achieved for most patients within 24 -48 hours.

\section{CONCLUSIONS}

- The ESPB is a strong potential tool as part of a multimodal analgesia regimen in lung transplantation.

REFERENCES:

1. Hopkins KG et al. J Adv Pract Oncol. 2015.

2. Gelzinis TA. JCVA 2018

3. Thabut G, Mal H. Journal of Thoracic Disease 2017.

4. McLean SR et al. JCVA 2018.

5. Forero $M$ et al. RAPM 2016.

6. Kelava M et al. JCVA 2018. 